\title{
A New Estimate of Building Floor Space in North America
}

Jay H. Arehart ${ }^{\mathrm{a}, \mathrm{b}^{*}}$, Francesco Pomponi ${ }^{\mathrm{b}}$, Bernardino D’Amico ${ }^{\mathrm{b}}$, and Wil V. Srubar III ${ }^{\mathrm{a}, \mathrm{c}}$

${ }^{a}$ Department of Civil, Environmental, and Architectural Engineering, University of Colorado Boulder,

ECOT 441 UCB 428, Boulder, Colorado 80309-0428 USA

${ }^{\mathrm{b}}$ Resource Efficient Built Environment Lab (REBEL), Edinburgh Napier University, Edinburgh, UK

${ }^{\mathrm{c}}$ Materials Science and Engineering Program, University of Colorado Boulder

${ }^{*}$ Corresponding Author, E: jay.arehart@,colorado.edu

\section{Number of pages: 6}

\section{Number of figures: 4}

\section{Number of tables: 1}

\section{Content summary}

To expand upon the methodology undertaken and results found within this study, we include the following appendices. For ease of use, we have included two files: Supplementary

Information_S1_S2_S3_S4.docx which contains additional plots and elaboration on methodology and Supplementary Information_S5_S6_S7_S8.xlsx which contains numerical results in the form of a datasheet.

\begin{tabular}{|c|c|c|}
\hline Code & Content & Filename \\
\hline S1 & $\begin{array}{l}\text { Identifying the Distance from } \\
\text { Road Threshold }\end{array}$ & $\begin{array}{l}\text { Supplementary Information_S1_S2_S3_S4.docx } \\
\text { (i.e., this file) }\end{array}$ \\
\hline S2 & $\begin{array}{l}\text { Sensitivity of Inter-Story Height } \\
\text { at the Building Stock Scale }\end{array}$ & $\begin{array}{l}\text { Supplementary Information_S1_S2_S3_S4.docx } \\
\text { (i.e., this file) }\end{array}$ \\
\hline S3 & $\begin{array}{l}\text { Predicted building height } \\
\text { distribution. }\end{array}$ & $\begin{array}{l}\text { Supplementary Information_S1_S2_S3_S4.docx } \\
\text { (i.e., this file) }\end{array}$ \\
\hline S4 & $\begin{array}{l}\text { Dataset Availability and Code } \\
\text { Repository }\end{array}$ & $\begin{array}{l}\text { Supplementary Information_S1_S2_S3_S4.docx } \\
\text { (i.e., this file) }\end{array}$ \\
\hline S5 & Street view validation & Supplementary Information_S3_S4_S5_S6.xlsx \\
\hline S6 & Floor space per capita by county & Supplementary Information_S3_S4_S5_S6.xlsx \\
\hline S7 & $\begin{array}{l}\text { Floor space estimate for different } \\
\text { distance to road threshold values } \\
\text { for the USA }\end{array}$ & Supplementary Information_S3_S4_S5_S6.xlsx \\
\hline S8 & $\begin{array}{l}\text { Floor space estimate for different } \\
\text { distance to road threshold values } \\
\text { for Canada }\end{array}$ & Supplementary Information_S3_S4_S5_S6.xlsx \\
\hline
\end{tabular}




\section{S1. Identifying the Distance from Road Threshold Value - Further Details}

The distance of a building's centroid to the nearest road varies significantly. $99 \%$ of building footprints identified in the Microsoft dataset are within $309 \mathrm{~m}$ of a road with $99.9 \%$ within $1052 \mathrm{~m}$. The different quantiles are show in Table S1.1. It is clear that the distribution of the distance from a road is non-linear, with some identified footprints being far from roads.

Table S1.1. Quantiles of the minimum distance to nearest road metric and footprint areas.

\begin{tabular}{|c|c|c|}
\hline Quantile & Distance (m) & Footprint Area $\mathbf{( m}^{\mathbf{2}} \mathbf{~}$ \\
\hline $1 \%$ & 7.8 & 25.6 \\
\hline $25 \%$ & 21.8 & 112.1 \\
\hline $50 \%$ & 27.6 & 169.2 \\
\hline $75 \%$ & 43.5 & 247.3 \\
\hline $99 \%$ & 309.3 & 1686 \\
\hline $99.9 \%$ & 1052 & 9115 \\
\hline
\end{tabular}

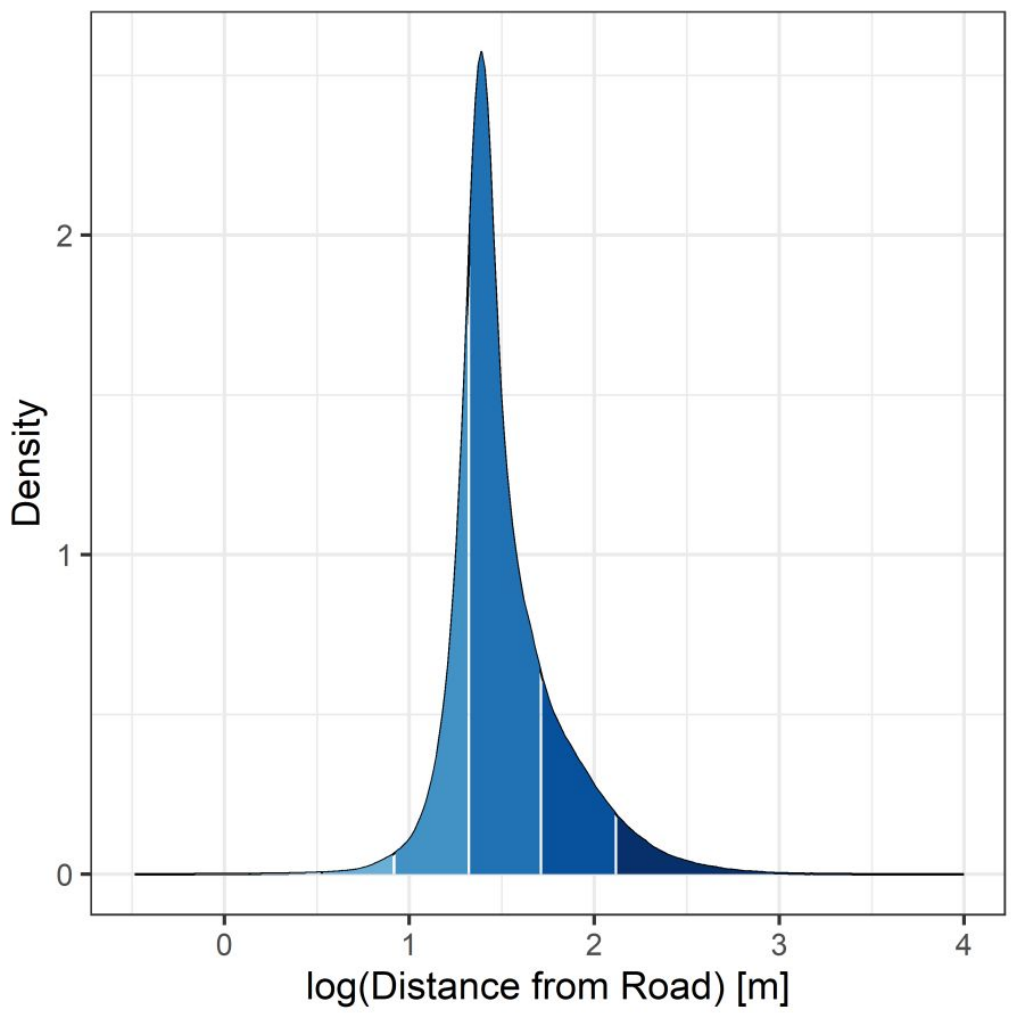

Figure S1.1. Density function, visualized by standard deviation, of the log-transform of distance from road.

To validate the fact that North American buildings in fact can be large distances from public roads, we visualize two cases in Figure S1.2. Many non-residential buildings (e.g., office buildings, shopping centers) have both large radii in terms of their building footprints in addition to being surrounded by large, private parking lots that are not included in the published roads datasets from the government. For example, the King of Prussia Mall has a distance of 224 meters from the nearest road. Similarly, in many rural parts of 
North America, buildings are located at the end of private driveways. For example, in rural Pennsylvania, a detached residence is noted at a distance of $235 \mathrm{~m}$ away from the publicly maintained roadway.

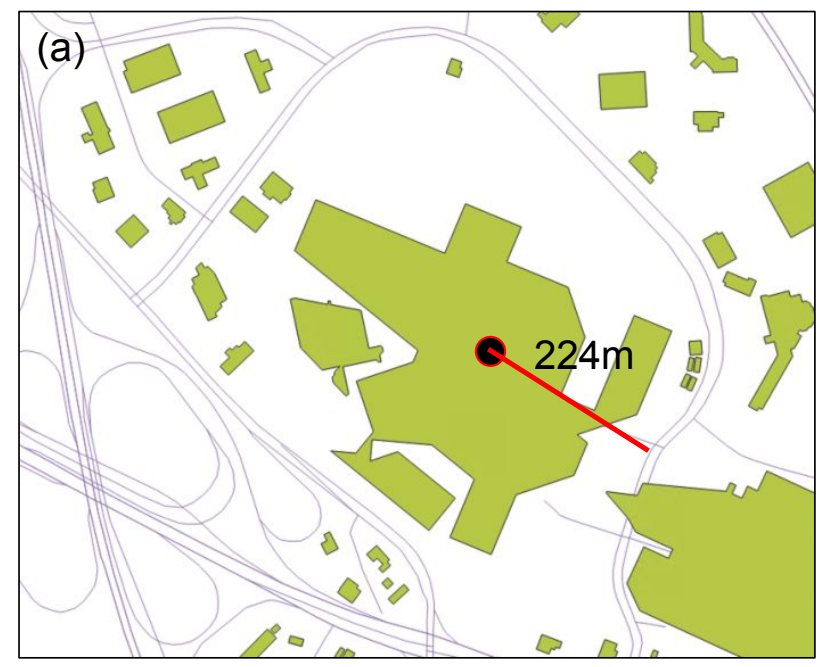

King of Prussia Mall

$(-75.39156,40.08821)$

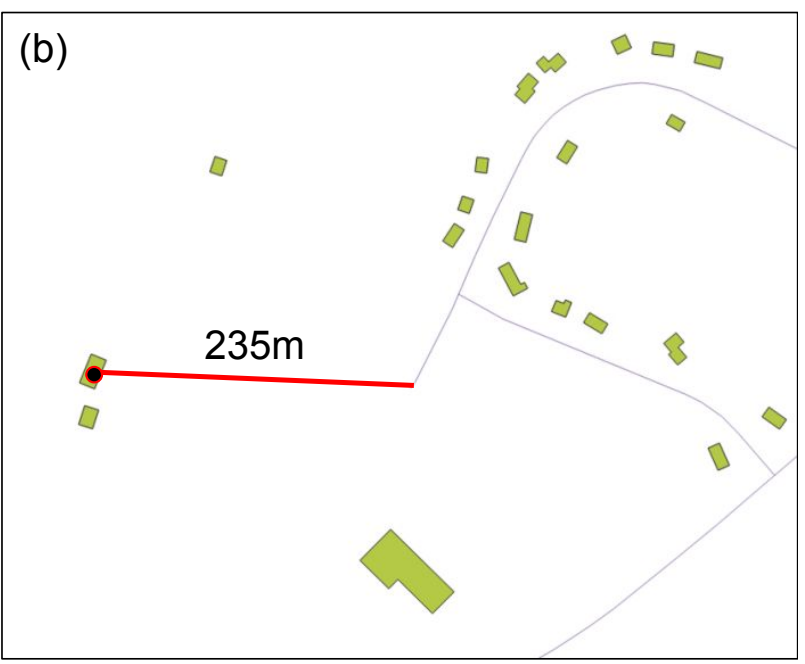

Private Driveway

$(-75.68536,39.94379)$

Figure S1.2. Examples of buildings of different sizes that are relatively large distances away from public roads. (a) shows the King of Prussia shopping mall which is a large commercial building surrounded by parking lots, while (b) shows a house at the end of a long, private driveway.

\section{S2. Sensitivity of Inter-Story Height at the Building Stock Scale}

For a single building, correctly estimating the number of stories is essential to accurately calculating the total floor space. Thus, knowing the building's interstory height (ISH) is imperative, yet this parameter is not known for each building considered herein. ISH has been recorded to typically range between $3 \mathrm{~m}$ and $6 \mathrm{~m}$ in building stocks ${ }^{1}$ with 3.5 to $3.65 \mathrm{~m}$ commonly used by the earthquake engineering community to model the dynamic behavior of buildings under seismic loads ${ }^{2,3}$. To investigate the sensitivity of our results to different assumptions on inter-story height (ISH) we analyzed a number of options to validate our assumption of ISH. For each of the following scenarios, we have taken 1 million building footprints and their associated height. Seven cases have been considered:

1. T3: all buildings have an ISH of $3 \mathrm{~m}$

2. T4: all buildings have an ISH of $4 \mathrm{~m}$

3. T5: all buildings have an ISH of $5 \mathrm{~m}$

4. T_nor: each building's ISH is randomly sampled from a normal distribution $(\mu=3.5, \sigma=0.5)$

5. $T \_\log$ : each building's ISH is randomly sampled form a lognormal distribution $(\mu=1.5, \sigma=0.5)$

6. T_uni: each building's ISH is randomly sampled from a uniform distribution $(\min =3, \max =5)$

7. T3.6: all buildings have an ISH of $3.6 \mathrm{~m}$

Of course, the assumption of ISH for each scenario has a varying degree of likelihood. Scenarios 1, 2, 3, and 7 are all impossible but they give us an idea of the total floor space when assuming a fixed number of ISH. The total floor space of these million building footprints is summarized in Figure S3. Scenarios 4, 5, 
and 6 are all possible but each contains oddities that we kept as they represented conservative assumptions, leading to an underestimate of floor area. For instance, for the lognormal and normal distributions there are (rare) cases where the randomly sampled ISH can be greater than the full height of that specific footprint. In these cases, we take the full height of the building as its ISH (i.e., assumption of a building to be single story). Note that this happens for very tall buildings at the right-hand tails of those two distributions (e.g., building heights $>12 \mathrm{~m}$ ) so surely considering those buildings to be only a single story is already an underestimation of floor area.

For the first cluster of 3 scenarios, we calculate an average ( $\operatorname{vvgl}$ ) that could be read as if we have 3 million buildings ( 1 million each with ISH of 3,4 , and $5 \mathrm{~m}$ ). This assumes that buildings with ISH of $5 \mathrm{~m}$ (e.g., warehouses, agricultural, mega-sheds etc.) are equally likely to exist than "normal" buildings and we know this is not the case. Thus, in reality, the shares would not be equal, and the actual average would be more dominated by the more popular ISH (e.g., 3 and $4 \mathrm{~m})$.

For the second cluster we calculate an average (avg2) that could be interpreted as if we have 3 million buildings ( 1 million with each ISH distribution). The three distributions have nothing to do with how we estimate in the first three scenarios and yet the averages are strongly aligned. An ISH of $3.6 \mathrm{~m}$ is what we have used in the present study and it is strongly aligned with all the averages calculated from the different assumptions. It is worth remembering once again that the built environment is dominated by buildings with ISH within a narrow range of $3.5-3.6 \mathrm{~m}$.

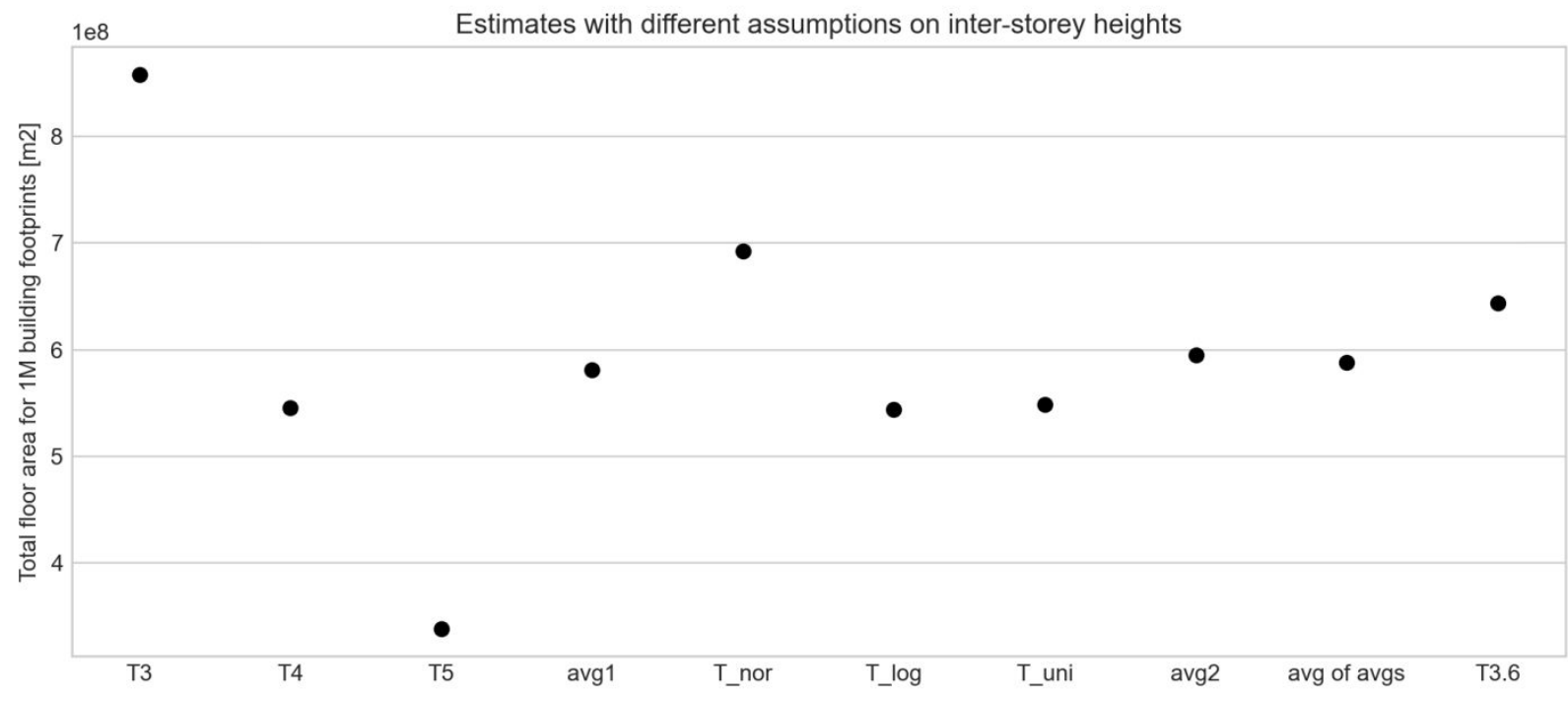

\begin{tabular}{|l|l|l|l|l|l|}
\hline T3: & ISH $=3 \mathrm{~m}$ & T_nor: & normal(mu=3.5, sigma=0.5) & avg3: & average(T3, T4, T5, T_nor, T_log, T_uni) \\
\hline T4: & ISH = 4m & T_log: & lognormal(mu=1.5, sigma=0.5) & T3.6: & ISH = 3.6m \\
\hline T5: & ISH = 5m & T_uni: & uniform(min=3, max=5) & & \\
\hline avg1: & average(T3, T4, T5) & avg2: & average(T_nor, T_log, T_uni) & & \\
\hline
\end{tabular}

Figure S2.1. Sensitivity analysis of interstory height (ISH) for different assumptions for a sample of 1 million buildings footprints. 


\section{S3. Predicted building height distribution.}

Figure S3.1 visualizes the distribution of building heights, predicted from the random forest regression model.

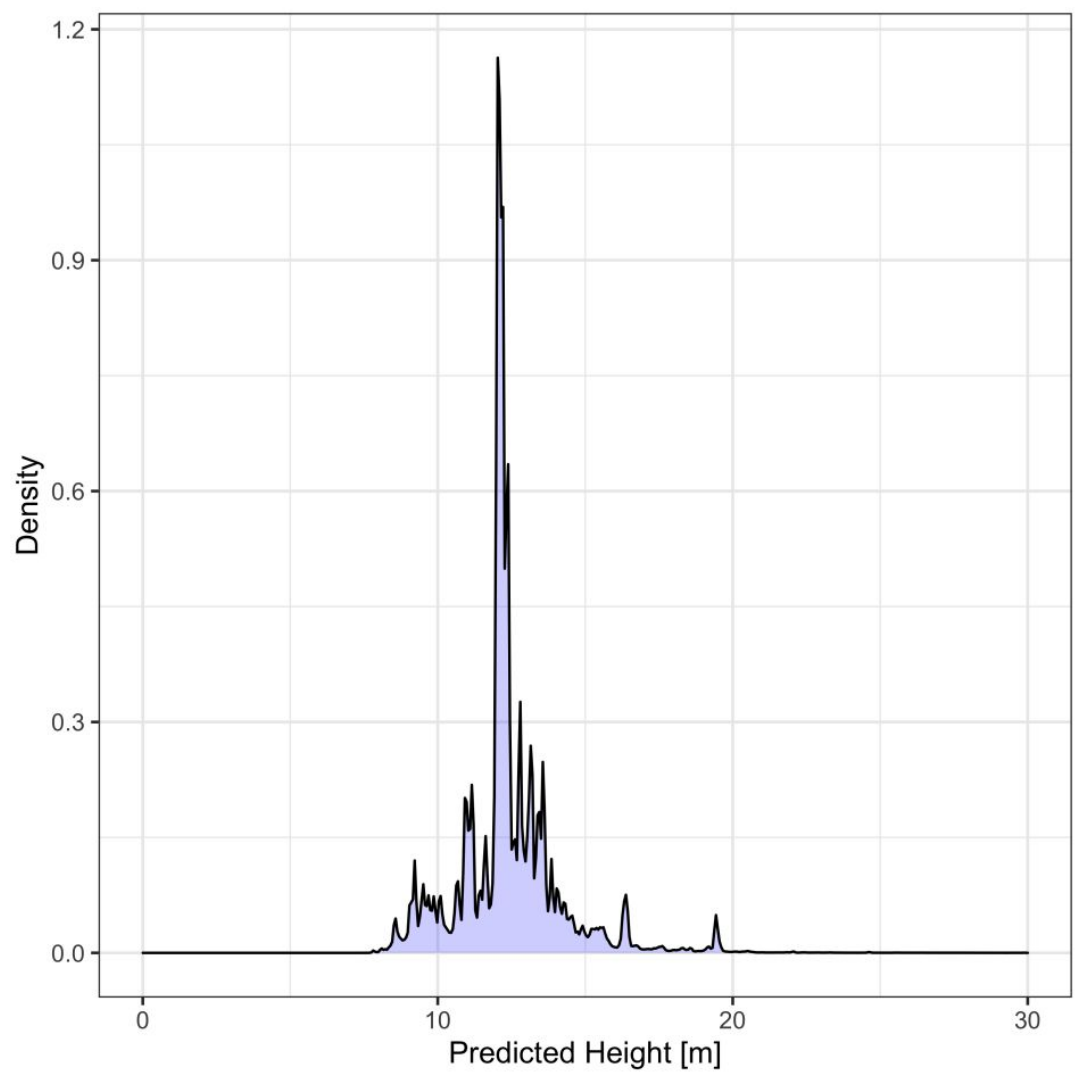

Figure S3.1. Distribution of predicted building height for all building footprints. Note that buildings taller than $30 \mathrm{~m}$ exists, but for clarity of the figure only heights less than $30 \mathrm{~m}$ are shown.

\section{S4. Dataset Availability and Code Repository}

The datasets used in this analysis are publicly available. For access to intermediate datasets (e.g., calculated geometric quantities), please contact the authors. Access to the model, and code used for the analysis can be found at: https://github.com/jayarehart/US Bldg Heights.

\section{References}

(1) Steadman, P.; Hamilton, I.; Evans, S. Energy and Urban Built Form: An Empirical and Statistical Approach. Building Research \& Information 2014, 42 (1), 17-31. https://doi.org/10.1080/09613218.2013.808140.

(2) Lin, J.-L.; Kek, M.-K.; Tsai, K.-C. Stiffness Configuration of Strongbacks to Mitigate Inter-Story Drift Concentration in Buildings. Engineering Structures 2019, 199, 109615. https://doi.org/10.1016/j.engstruct.2019.109615.

(3) Ramamoorthy, S. K.; Gardoni, P.; Bracci, J. M. Seismic Fragility and Confidence Bounds for Gravity Load Designed Reinforced Concrete Frames of Varying Height. J. Struct. Eng. 2008, 134 (4), 639650. https://doi.org/10.1061/(ASCE)0733-9445(2008)134:4(639). 